\title{
Stable and Low Prevalence of Transmitted HIV Type 1 Drug Resistance Despite Two Decades of Antiretroviral Therapy in Hong Kong
}

\author{
K.H. Wong, W.K. Chan, W. W. Yam, ${ }^{2}$ J.H.K. Chen, ${ }^{2}$ F.R. Alvarez-Bognar, and K.C.W. Chan ${ }^{1}$
}

\begin{abstract}
Transmitted HIV resistance is of both clinical and public health importance. Baseline genotypic resistance testing was performed for HIV-1-infected treatment-naive patients who were newly diagnosed between 2003 and 2007 and attended the government HIV clinic in Hong Kong. International AIDS Society-USA mutation figures and the Stanford resistance interpretation algorithm were used to identify resistance mutations and drug susceptibility, respectively. The pattern and factors associated with resistance were examined. The presence of one or more IAS-USA resistance mutations was found in 26 (3.6\%) of 731 patients over the 5-year study period. Overall, protease inhibitor (PI) resistance mutations were most common (16), followed by nucleoside reverse transcriptase inhibitors (NRTIs) (8) and nonnucleoside reverse transcriptase inhibitors (NNRTIs) (3). Resistance to drugs in one, two, and three classes was present in $25(3.4 \%), 1(0.1 \%)$, and 0, respectively. Seventy-eight $(10.7 \%)$ had strains of reduced susceptibility, as predicted by the Stanford algorithm to display at least low-level resistance to one or more drugs of the three classes. Intermediate or high-level resistance was found in $1.6 \%$ overall, and in descending order for NRTIs, PIs, and NNRTIs. There was no temporal trend of increase in resistance. Sex between men, Chinese ethnicity, and lower baseline CD4 were associated with harboring resistant strains as elucidated by either method. We conclude that transmitted HIV-1 drug resistance is uncommon in up to two decades of antiretroviral therapy in Hong Kong. The situation has to be continually monitored for any change in significance.
\end{abstract}

\section{Introduction}

$\mathbf{T}$ HE ADVENT OF HIGHLY ACTIVE ANTIRETROVIRAL THERAPY (HAART) has turned HIV disease into a chronic medical condition with greatly reduced AIDS-related morbidity and mortality. ${ }^{1-3}$ Nevertheless, HAART is not a panacea, for despite treatment, HIV-infected patients still have a shorter life expectancy than people in the general population. ${ }^{4}$ Moreover, as with other antiinfectives, resistance may have existed before HAART is started or may emerge during the course of treatment. Drug resistance is one major factor contributing to HIV treatment failure. ${ }^{5}$ It is hence prudent to tackle the resistance issue if we are to achieve optimal HIV management and care.

Resistance testing is now a standard component of laboratory diagnosis and monitoring of HIV infection. ${ }^{6}$ Together with CD4 and HIV-1 viral load measurements, these three specific tools greatly aid day-to-day medical management of
HIV / AIDS patients. As early as 2000, the International AIDS Society (IAS)-USA recommended using the HIV resistance test in patients with chronic HIV infection before initiation of antiretroviral therapy. ${ }^{7}$ The rationale is that baseline resistance may reduce treatment efficacy, thus requiring the firstline regimen to be adjusted accordingly. Resistance sampling should be done as close to the time of infection as possible, as resistant viruses back-mutate to wild-type genotypes with time. Genotypic resistance testing (GRT) is often the preferred test to phenotypic assay because of its simpler laboratory procedures and stronger evidence of virologic benefits and cost effectiveness. $^{8}$

The usefulness of application of resistance testing in treatment-naive subjects may, however, differ from place to place due to the varied epidemiology of transmitted resistance. The HIV clinic at the Integrated Treatment Centre (ITC) of the Hong Kong Department of Health has introduced GRT in the baseline workup of newly diagnosed patients since 2003. We

\footnotetext{
${ }^{1}$ Integrated Treatment Centre, Special Preventive Programme, Centre for Health Protection, Department of Health, Hong Kong.

${ }^{2}$ Department of Microbiology, Queen Mary Hospital, The University of Hong Kong, Hong Kong.
} 
set out to examine the prevalence of transmitted resistance, its temporal trend, and associated factors in a cohort of new patients seen over a period of 5 years.

\section{Materials and Methods}

\section{Study subjects}

The ITC is the largest care provider to adults living with HIV in Hong Kong. Our patients are referred from a variety of sources, including hospitals, sexually transmitted infections clinics, and voluntary HIV testing sites. Preceded by monotherapy with the first use of zidovudine in 1987 and afterward by dual therapy, HAART became the standard antiretroviral treatment in late $1996 .^{9}$ About $85 \%$ of patients with AIDS and/or CD $4<200$ cells / $\mu$ l were on HAART. ${ }^{10}$ Treatment and prophylaxis of opportunistic infections were prescribed as appropriate per international recommendations. The AIDS case definition for adults and adolescents in Hong Kong is adopted from the US CDC 1993 definition, with the following modifications: (1) disseminated penicilliosis is included as an AIDS-defining illness (ADI), (2) pulmonary or cervical lymph node tuberculosis is counted as an ADI only if the CD4 count is $<200$ cells / $\mu$ l, and (3) a CD 4 count of $<200$ cells / $\mu$ l alone is not considered as AIDS. ${ }^{11}$ In the present study, we analyzed patients who were diagnosed as HIV-1 positive between 2003 and 2007 and had been antiretroviral naive before a baseline genotypic resistance test was done at our clinic. The testing forms a surveillance effort to monitor HIV resistance in the locality. We looked at all patients, including those with recent infections as defined by a prior negative HIV antibody test or seroconversion illness within 1 year of HIV diagnosis.

\section{Resistance test and interpretation}

The genotypic assay is the resistance test employed in our clinic. The laboratory procedures of HIV-1 RNA extraction, amplification, and sequencing have been previously described. ${ }^{12}$ In addition to an FDA-approved ViroSeq ${ }^{\mathrm{TM}}$ system, an in-house system was also used. We had demonstrated comparable performance in terms of sensitivity, specificity, and detection of resistance mutations on a wide range of HIV1 subtypes for this in-house genotyping system. ${ }^{13}$ Two methods were used to examine the harboring of resistant strains in our study patients. First, we assessed the presence of resistance mutations (RTI resistance associated or major PI resistance associated) against the Mutations Figures published by the IAS-USA Drug Mutations Group (December 2008 update, http://iasusa.org). Second, the pol sequences were analyzed using the Stanford University HIV Drug Resistance Database HIVdb program (version 4.2.6 http:// hivdb.stanford.edu). Under the Stanford algorithm, there are five interpretation results of susceptibility to each drug, namely susceptible, potential low-level resistance, low-level resistance, intermediate resistance, and high-level resistance. In this study, HIV-1 strains predicted to exhibit low-level or greater resistance per Stanford interpretation were counted as having reduced drug susceptibility and thus resistance. We restricted data analysis to the three mainstay drug classesnucleoside reverse transcriptase inhibitors (NRTIs), nonnucleoside reverse transcriptase inhibitors (NNRTIs), and protease inhibitors (PIs), as the newer classes, e.g., fusion in- hibitor and integrase inhibitor, had not been used by our clinic through the study period.

\section{Statistical analysis}

Differences in categorical items such as proportion of resistant strains were examined by logistic regression tests while continuous variables were analyzed by independent $t$ test. Cox proportional hazards logistic regression of univariate and multivariate analyses was used to study demographic and HIV infection variables that may be associated with resistance. Their crude and adjusted hazard ratios and 95\% confidence intervals were calculated. We used SPSS (version 11.0) for all statistical analyses. All tests of significance were two-sided, and a $p<0.05$ was considered to be statistically significant.

\section{Results}

During the 5-year study period, a total of 865 antiretroviralnaive patients with newly diagnosed HIV infection attended our clinic. Of them, 134 (15.5\%) were excluded from analysis because of the lack of baseline genotypic resistance testing results. Table 1 shows the patient and HIV disease characteristics of the 731 study subjects. The majority of the patients were male (87\%), aged between 30 and 49 years $(58 \%)$, and had acquired HIV via sexual contact $(86.6 \%)$. HIV-1 subtype B and CRF01_AE accounted for $42.4 \%$ and $43.1 \%$, respectively. Cases of recent infection significantly differed from nonrecent cases in being younger, more often infected with the B subtype, and less likely to be AIDS patients, and having a higher baseline CD4 cell count. There were more men who have sex with men (MSM) among recently infected patients, but not with statistical significance. There was no significant change in disease stage of the patients in terms of presenting CD4 or AIDS over the study period.

Table 2 shows the resistance profile of the study subjects. Overall, $26(3.6 \%)$ had at least one resistance mutations per IAS-USA figures. Each year, one to eight patients (0.8-6.4\%) were detected as having baseline resistance mutations. Among all patients harboring resistant viruses, PI resistance was the commonest and was present in 16 subjects, with M46L (seven), M46I (three), and L33F (three) being the most frequent mutations. NRTI came second in class with eight patients showing resistance; thymidine analogue mutations (eight) were much commoner than M184V (one). Baseline NNRTI resistance was present in only three $(0.4 \%)$ subjects. Taken together, resistance to one and two classes of antivirals was found in $25(3.4 \%)$ and $1(0.1 \%)$. None of the patients had three-class resistance.

Based on the Stanford HIVdb algorithm, drug susceptibility was possibly reduced in $78(10.7 \%)$ of subjects over the 5year period (Fig. 1). Most of the reduced drug susceptibility cases were of low-level resistance and only $12(1.6 \%)$ were intermediate or high-level resistance. The frequency of resistance by class per Stanford analysis is different from that based on IAS-USA mutation figures. Considering all cases with reduced drug susceptibility, resistance to NNRTI was most common and was found in $37(5.1 \%)$, followed by 24 (3.3\%) with NRTI resistance and 22 (3.0\%) with PI resistance. If only strains with intermediate or high-level resistance were considered, the NRTI class ranked first at six $(0.8 \%)$ and the NNRTI class came last at two $(0.3 \%)$. Three $(0.4 \%)$ patients 
TAble 1. Demographic ANd baseline HIV disease Characteristics

\begin{tabular}{|c|c|c|c|c|}
\hline & All & Recent infection ( $<1$ year) & Chronic infection & $\mathrm{p}$ Value \\
\hline Number of patients & 731 & $114(15.6 \%)$ & $617(84.4 \%)$ & \\
\hline Sex, $n(\%)$ & & & & 0.395 \\
\hline Male & $636(87.0)$ & $102(89.7)$ & $534(86.6)$ & \\
\hline Female & $95(13.0)$ & $12(10.5)$ & $83(13.5)$ & \\
\hline Ethnicity, $n(\%)$ & & & & 0.262 \\
\hline Chinese & $560(76.6)$ & $92(80.7)$ & $468(75.9)$ & \\
\hline Non-Chinese & $171(23.4)$ & $22(19.3)$ & $149(24.2)$ & \\
\hline Age, year & & & & 0.023 \\
\hline Mean & 39.1 & 36.6 & 39.5 & \\
\hline Median & 36.3 & 34.7 & 36.9 & \\
\hline HIV exposure category, $n(\%)$ & & & & 0.908 \\
\hline Heterosexual & $311(42.5)$ & $37(32.5)$ & $274(44.4)$ & \\
\hline Sex between men & $322(44.1)$ & $74(64.9)$ & $248(40.2)$ & \\
\hline Other & $98(13.4)$ & $3(2.6)$ & $95(15.4)$ & \\
\hline HIV-1 subtype, $n$ (\%) & & & & $<0.001$ \\
\hline B & $310(42.4)$ & $70(61.4)$ & $240(38.9)$ & \\
\hline CRF01_AE & $315(43.1)$ & $34(29.8)$ & $281(45.5)$ & \\
\hline Other & $82(11.2)$ & $5(4.4)$ & 77 (12.5) & \\
\hline AIDS, $n(\%)$ & $155(21.2)$ & $1(0.9)$ & $154(25.0)$ & $<0.001$ \\
\hline CD4 in cells $/ \mu \mathrm{l}$, median (range) & $247(0-1423)$ & $405.5(20-942)$ & $208(0-1423)$ & $<0.001$ \\
\hline Viral load in log copies/ml, mean (range) & $4.95(2.6-6.86)$ & $4.91(2.6-6.34)$ & $4.97(2.6-6.86)$ & 0.029 \\
\hline
\end{tabular}

Table 2. Resistance Profile by Presence of Major Mutation PER IAS-USA Mutation Figures (2003-2007)

\begin{tabular}{|c|c|c|}
\hline Mutation & $\begin{array}{c}\text { Frequency } \\
\text { in all patients, } \\
\mathrm{n}(\%)\end{array}$ & $\begin{array}{c}\text { Frequency in patients } \\
\text { with drug-resistant } \\
\text { strains, } \%\end{array}$ \\
\hline \multicolumn{3}{|l|}{ All drugs } \\
\hline $\begin{array}{l}\text { Any (NRTI, } \\
\text { NNRTI, PI) }\end{array}$ & $26(3.6)$ & \\
\hline 1 -class & $25(3.4)$ & 96.2 \\
\hline 2-class & $1(0.1)$ & 3.9 \\
\hline 3-class & $0(0.0)$ & 0.0 \\
\hline \multicolumn{3}{|l|}{ NRTI } \\
\hline Any & $8(1.1)$ & 30.8 \\
\hline 1 & $6(0.8)$ & 23.1 \\
\hline$\geq 2$ & $2(0.3)$ & 7.7 \\
\hline$\overline{\mathrm{M}} 41 \mathrm{~L}$ & $2(0.3)$ & 7.7 \\
\hline D67N & $2(0.3)$ & 7.7 \\
\hline M184V & $1(0.1)$ & 3.9 \\
\hline M184I & $1(0.1)$ & 3.9 \\
\hline K219Q & $4(0.5)$ & 15.4 \\
\hline \multicolumn{3}{|l|}{ NNRTI } \\
\hline Any & $3(0.4)$ & 11.5 \\
\hline 1 & $3(0.4)$ & 11.5 \\
\hline$\geq 2$ & $0(0.0)$ & 0.0 \\
\hline$\overline{\mathrm{K}} 103 \mathrm{~N}$ & $1(0.1)$ & 3.8 \\
\hline V108I & $1(0.1)$ & 3.8 \\
\hline Y181C & $1(0.1)$ & 3.8 \\
\hline \multicolumn{3}{|l|}{ PI } \\
\hline Any & $16(2.2)$ & 61.5 \\
\hline 1 & $15(2.1)$ & 57.7 \\
\hline$\geq 2$ & $1(0.1)$ & 3.9 \\
\hline$\overline{\mathrm{L}} 33 \mathrm{~F}$ & $3(0.4)$ & 11.5 \\
\hline M46I & $3(0.4)$ & 11.5 \\
\hline M46L & $7(1.0)$ & 26.9 \\
\hline Q58E & $2(0.3)$ & 7.7 \\
\hline V82A & $1(0.1)$ & 3.9 \\
\hline L90M & $1(0.1)$ & 3.9 \\
\hline
\end{tabular}

harbored viruses that had reduced susceptibility to two classes of drugs, but only of low-level resistance with none being intermediate or high-level resistant.

Five patients with IAS-USA mutations did not have reduced susceptibility by Stanford while 57 subjects with reduced susceptibility did not show IAS-USA mutations. The commonest mutations of these patients leading to reduced susceptibility were for the reverse transcriptase gene: V179D (22), T69N (10), A98G (5), V179E (4), and for the protease gene: V82I (4), G16E (3), K20I (2), L10I (2), and T74S (2). Many of them are minor mutations. We examined treatment response among patients with CD $4<200$ cells / $\mu$ l and found that $100 \%$ $(1 / 1), 100 \%(8 / 8)$, and $90.3 \%(28 / 31)$ of subjects with IASUSA mutations alone, both IAS-USA mutations and reduced susceptibility, and reduced susceptibility alone, respectively, had undetectable viral load at 12 months post-HAART.

We performed factor analysis of resistant cases as identified from both the IAS-USA and Stanford methods. Univariate and multivariate analyses using Cox proportional hazards regression showed that sex between men as the exposure category was associated with a higher likelihood of the presence of IAS-USA mutations (Table 3). Recent infection, age, gender, ethnicity, HIV-1 subtype, baseline CD4, and viral load were not significant factors. Also, year of HIV diagnosis was not an associated variable and there was no definite time trend of resistance. Interestingly, non-Chinese and baseline CD4 $\geq 200$ cells / $\mu$ l were associated with a lower risk of reduced drug susceptibility by the Stanford algorithm on both univariate and multivariate analyses (Table 4). Nonsexual HIV exposure was a factor in univariate but not multivariate analysis. Again, year of HIV diagnosis was not a significant variable. There was no temporal pattern in reduced susceptibility to any drugs by the Stanford algorithm during the study period (Fig. 1). Moreover, none of the factors examined was significant if we excluded low-level resistance cases and analyzed only cases of intermediate or high-level resistance. 


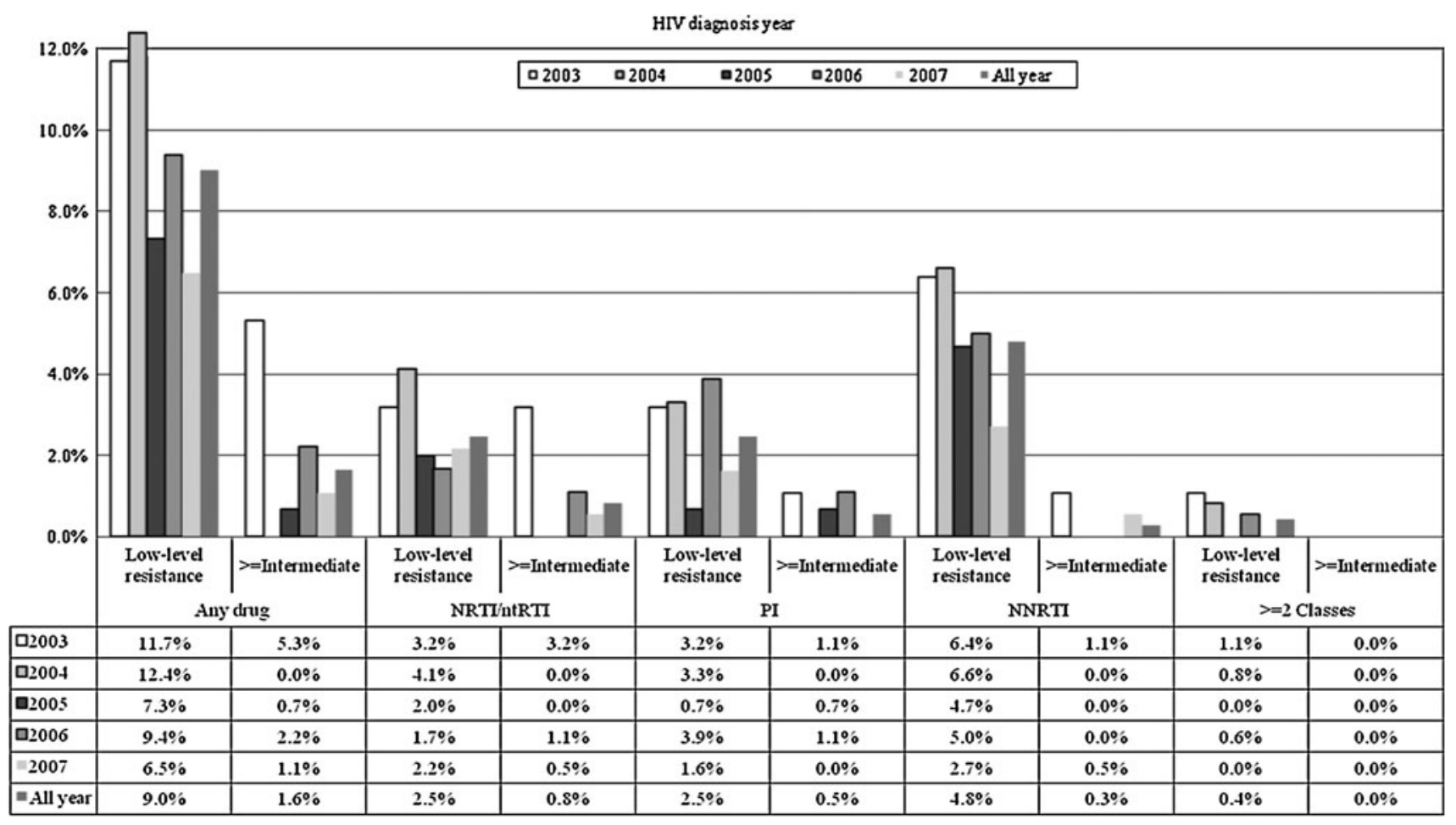

FIG. 1. Proportion of patients with predicted reduced drug susceptibility by Stanford HIVdb algorithm (2003-2007).

\section{Discussion}

In this study, we examined the prevalence and factors of transmitted HIV-1 drug resistance in Hong Kong, two decades into the availability of the first antiretroviral drug zidovudine in 1987 and 6-10 years after full application of HAART as the local standard in 1997. Being the largest HIV clinic in Hong Kong, our findings can shed light on the territory-wide situation. Indeed, our study population was similar in basic demographics and HIV disease characteristics to all reported infections in Hong Kong during the period, except that there was a smaller proportion of female and non-Chinese patients (data not shown). Published studies on HIV resistance were mostly from Western developed countries in the past. However, reports are now increasingly being made in other parts of the world, including Asia. ${ }^{14-17}$ Our study population, comprising mostly Chinese, contributes to the understanding of resistance in the Asia Pacific region. Also, unlike some previous Asian reports with limited treatment accessibility, ${ }^{14,16}$ our findings can provide information concerning transmitted resistance against a background of high treatment coverage beyond Western countries.

We found a low frequency of drug resistance at about 3\% in treatment-naive patients over the 5-year study period, and without a discernible rising trend over time by two methods of resistance determination. Furthermore, most of these patients harbored strains resistant to one drug class only, as dual-class resistance was rare at $<0.2 \%$ and three-class resistance was nonexistent. The frequency of transmitted resistance ranged from $5 \%$ to $10 \%$ in North America and European countries ${ }^{18-20}$ but had been reported to be as high as 20$25 \%{ }^{21}$ Data from 1996 to 2005 of the Swiss HIV cohort sug- gested that transmission of drug resistance in the setting of easy access to antiretroviral treatment can remain stable and be kept at a low level. ${ }^{18}$ We believe that good drug adherence contributed to the low resistance found in this study, as we previously reported $<4 \%$ of our patients had less than $95 \%$ adherence. ${ }^{22}$ Improved treatment efficacy with more patients in virologic success, which reduces the likelihood of transmission of resistant strains, has also been postulated to contribute to the stable or decreasing transmitted resistance in Western Europe. ${ }^{19,23}$ Noteworthy, our study population did not have an increase in NNRTI-resistant virus transmission over time, in contrast to several overseas reports. ${ }^{18-20,24,25}$ This is most encouraging as single resistance mutation can effectively render the whole class of NNRTIs useless, with the possible exception of etravirine.

The importance of assessing and monitoring for transmitted HIV-1 drug resistance cannot be overstated, as transmission of drug-resistant HIV-1 has been reported in many countries with access to antiretroviral treatment, albeit at different levels. The World Health Organisation (WHO) recommended that the capability of resistance testing be developed even in resource-constrained settings, using a threshold survey of recently infected individuals as a minimum-resource strategy to evaluate transmitted resistance. ${ }^{26}$ Such a recommendation should be adopted or taken note of by all places instituting antiretroviral therapy, which is quickly becoming the standard due to the global scale up of treatment. The value of monitoring transmitted resistance is to guide and maximize the success of first-line treatment regimens designed under a public health approach as advocated by the WHO. The prevalence of transmitted resistance is set at thresholds of $5 \%$ and $15 \%$, representing low and high levels of resistance, respectively. 
Table 3. Univariate and Multivariate Analyses of Factors Associated with the Presence of Resistance Mutation to Any Drug Class (2003-2007)

\begin{tabular}{|c|c|c|c|}
\hline Variable & $\mathrm{n}$ & Crude HR (95\% CI) & Adjusted HR (95\% CI) \\
\hline \multicolumn{4}{|l|}{ Age, year } \\
\hline$<40$ & 16 & 1 & \\
\hline$\geq 40$ & 10 & $1.25(0.57-2.78)$ & \\
\hline \multicolumn{4}{|l|}{ Sex } \\
\hline Male & 24 & 1 & \\
\hline female & 2 & $0.68(0.16-2.89)$ & \\
\hline \multicolumn{4}{|l|}{ Ethnicity } \\
\hline Chinese & 23 & 1 & \\
\hline Non-Chinese & 3 & $0.26(0.08-0.88)$ & \\
\hline \multicolumn{4}{|c|}{ HIV exposure category } \\
\hline Heterosexual & 7 & 1 & 1 \\
\hline Sex between men & 18 & $3.04(1.25-7.37)$ & $3.04(1.25-7.37)$ \\
\hline Other & 1 & $0.29(0.04-2.39)$ & $0.29(0.04-2.39)$ \\
\hline \multicolumn{4}{|l|}{ HIV-1 subtype, $n$ (\%) } \\
\hline B & 15 & 1 & \\
\hline CRF01_AE & 10 & 0.48 (0.21-1.09) & \\
\hline Other & 1 & $0.21(0.03-1.56)$ & \\
\hline \multicolumn{4}{|l|}{ AIDS } \\
\hline Yes & 3 & 1 & \\
\hline No & 23 & $2.63(0.79-8.76)$ & \\
\hline \multicolumn{4}{|c|}{ Baseline CD4 in cells / $\mu$ l } \\
\hline$<200$ & 11 & 1 & \\
\hline$\geq 200$ & 15 & $1.07(0.49-2.33)$ & \\
\hline \multicolumn{4}{|c|}{ Baseline viral load in copies $/ \mathrm{ml}$} \\
\hline$<5 \log$ & 17 & 1 & \\
\hline$\geq 5 \log$ & 9 & $0.57(0.25-1.27)$ & \\
\hline \multicolumn{4}{|l|}{ Recent infection } \\
\hline No & 21 & 1 & \\
\hline Yes & 5 & $1.50(0.57-3.99)$ & \\
\hline \multicolumn{4}{|l|}{ Year of HIV diagnosis } \\
\hline 2003 & 6 & 1 & \\
\hline 2004 & 1 & $0.15(0.18-1.27)$ & \\
\hline 2005 & 6 & $0.86(0.28-2.66)$ & \\
\hline 2006 & 8 & $0.84(0.29-2.45)$ & \\
\hline 2007 & 5 & $0.47(0.14-1.55)$ & \\
\hline
\end{tabular}

In the absence of a universal consensus on the interpretation of genotypic resistance results, the frequency of transmitted resistance would depend on the definition and method employed in a particular study to gauge HIV-1 drug resistance. $^{27}$ We found that transmitted PI resistance presented more frequently than NRTI resistance in our patient population per the presence of IAS-USA mutations. Even though one study supported this finding, ${ }^{28}$ other reports had shown the reverse, that NRTI resistance was the most frequently found. ${ }^{29,30}$ Our clinic has consistently used PI as the preferred $(>70 \%)$ regimen for treatment initiation. In fact, $\mathrm{M} 46 \mathrm{I} / \mathrm{L}$ mutations, which confer resistance to indinavir, accounted for half of the cases with PI resistance. Such findings could be related to our frequent use of indinavir, which is well known to cause intolerance, in the early days of the HAART era. Therefore, the uncommon use of NNRTIs in the first regimen contributed to the relative rarity of significant NNRTI mutations. Nonetheless, due to methodological variation in data interpretation, it is not appropriate to directly compare our prevalence findings with those collected in other studies. It is interesting but prospective that the resistance results differed between mutation figures and the drug susceptibility algorithm in our study. However, the frequency of resistance as informed by the Stanford algorithm was similar to consensus mutation findings, if only intermediate or above level of reduced susceptibility was considered. Indeed, our clinic experience showed that treatment efficacy was seldom compromised in patients with just low-level resistance by the Stanford analysis, supporting the greater clinical relevance of using an intermediate or higher level of reduced susceptibility. We suggest taking reference of both IAS-USA mutation figures and the Stanford algorithm testing for guiding treatment as they are widely used methods of genotypic resistance interpretation.

Factor analysis also yielded different findings of resistance association for IAS-USA mutation figures and drug susceptibility testing by Stanford. On multivariate analysis, while HIV exposure category is a risk factor by mutation figures, Chinese ethnicity and lower baseline CD4 were associated with transmitted resistance by the Stanford algorithm. The fact that resistant cases were few could have limited the analysis of factors associated with the occurrence of resistance, particularly when only intermediate or above level resistance was considered. Our findings did not suggest an increasing trend of transmitted resistance overall or a higher prevalence in recent infections; the true extent of the latter 
Table 4. Univariate and Multivariate Analyses of Factors Associated with Reduced Susceptibility to Any Drug Class (Low or Above the Level of Resistance) by the Stanford Algorithm (2003-2007)

\begin{tabular}{|c|c|c|c|}
\hline Variable & $\mathrm{n}$ & Crude HR (95\% CI) & Adjusted HR $(95 \%$ CI) \\
\hline \multicolumn{4}{|l|}{ Age, year } \\
\hline$<40$ & 46 & 1 & \\
\hline$\geq 40$ & 32 & $1.48(0.93-2.34)$ & \\
\hline \multicolumn{4}{|c|}{ 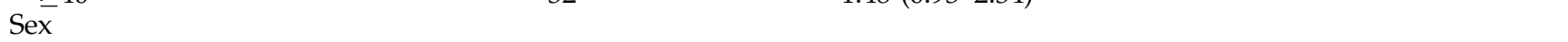 } \\
\hline Male & 69 & & \\
\hline female & 9 & $1.05(0.53-2.12)$ & \\
\hline \multicolumn{4}{|l|}{ Ethnicity } \\
\hline Chinese & 64 & 1 & 1 \\
\hline Non-Chinese & 14 & $0.37(0.20-0.68)$ & $0.53(0.28-0.99)$ \\
\hline \multicolumn{4}{|c|}{ HIV exposure category } \\
\hline Heterosexual & 37 & 1 & \\
\hline Sex between men & 35 & $1.27(0.79-2.05)$ & \\
\hline Other & 6 & $0.32(0.13-0.75)$ & \\
\hline \multicolumn{4}{|l|}{ HIV-1 subtype, $n(\%)$} \\
\hline B & 33 & 1 & \\
\hline CRF01_AE & 35 & $0.63(0.38-1.04)$ & \\
\hline Other & 8 & $0.67(0.31-1.46)$ & \\
\hline \multicolumn{4}{|l|}{ AIDS } \\
\hline Yes & 21 & 1 & \\
\hline No & 57 & $0.98(0.58-1.63)$ & \\
\hline \multicolumn{4}{|l|}{ CD4 in cells $/ \mu \mathrm{l}$} \\
\hline$<200$ & 47 & 1 & 1 \\
\hline$\geq 200$ & 31 & $0.55(0.35-0.86)$ & $0.55(0.35-0.89)$ \\
\hline \multicolumn{4}{|c|}{ Viral load in copies/ml } \\
\hline$<5 \log$ & 37 & 1 & \\
\hline$\geq 5 \log$ & 41 & $1.13(0.72-1.77)$ & \\
\hline \multicolumn{4}{|l|}{ Recent infection } \\
\hline No & 71 & 1 & \\
\hline Yes & 7 & $0.66(0.30-1.43)$ & \\
\hline \multicolumn{4}{|l|}{ Year of HIV diagnosis } \\
\hline 2003 & 16 & 1 & \\
\hline 2004 & 15 & $0.94(0.46-1.93)$ & \\
\hline 2005 & 12 & $0.71(0.33-1.53)$ & \\
\hline 2006 & 21 & 0.97 (0.49-1.9) & \\
\hline 2007 & 14 & $0.58(0.28-1.22)$ & \\
\hline
\end{tabular}

might have been missed in some patients without a previous HIV test or seroconversion illness. In all, the low frequency of transmitted resistance and the few associated factors identified make selective identification of at-risk patients for pretreatment resistance testing impossible. To improve patient management and the yield of surveillance, our findings support testing of all clinic patients. Whether resistance is persistently higher in certain subgroups requires further monitoring and studies.

Our study had several limitations. We could have overestimated the transmitted resistance if some resistant patients had actually received treatment before and then developed resistance. But this was unlikely as all subjects were our clinic patients and we had their detailed history, unlike that of a pure laboratory setting, which may not have sufficient clinical information on the cases. On the other hand, some resistant cases could have their viruses reverted to wild types at the time of HIV diagnosis after long-standing infection. Underestimation due to this factor is, however, unavoidable unless only acute or recent infections are studied. Also, its effect, if any, would likely be similar throughout the study period as there was no evidence of increasing late presentations as reflected by the presenting CD4 or AIDS diagnosis. Consistent sampling for baseline GRT of newly diagnosed patients at our clinic is a strength of our findings.

In summary, the present study suggests a stable and low level of transmitted drug resistance in HIV-infected patients attending the government HIV clinic despite two decades of antiretroviral treatment provision in Hong Kong. Although this observation for the moment is reassuring, transmitted HIV resistance has to be continually monitored to inform public health epidemiology as well as drug treatment at an individual care level.

\section{Acknowledgments}

The work was supported by the AIDS Trust Fund of the Hong Kong Special Administrative Region Government (Grant MSS-099R and 155R). The authors are grateful to all staff of the Integrated Treatment Centre for their dedicated care of the patients. The opinions and assertions contained herein are the private views of the authors and do not necessarily reflect those of the Centre for Health Protection, Hong Kong Department of Health.

\section{Author Disclosure Statement}

No competing financial interests exist. 


\section{References}

1. Palella FJ Jr, Delaney KM, Moorman AC, et al.: Declining morbidity and mortality among patients with advanced human immunodeficiency virus infection. HIV Outpatient Study Investigators. N Engl J Med 1998;338:853-860.

2. Murphy EL, Collier AC, Kalish LA, et al.: Viral Activation Transfusion Study Investigators. Highly active antiretroviral therapy decreases mortality and morbidity in patients with advanced HIV disease. Ann Intern Med 2001;135:17-26.

3. Wong KH, Chan KCW, and Lee SS: Delayed progression to death and to AIDS in a Hong Kong cohort of patients with advanced HIV disease during the era of highly active antiretroviral therapy. Clin Infect Dis 2004;39:853-860.

4. Lohse N, Hansen AB, Pedersen G, et al.: Survival of persons with and without HIV infection in Denmark, 1995-2005. Ann Intern Med 2007;146:87-95.

5. Kuritzkes DR, Lalama CM, Ribaudo HJ, et al.: Preexisting resistance to nonnucleoside reverse-transcriptase inhibitors predicts virologic failure of an efavirenz-based regimen in treatment-naive HIV-1-infected subjects. J Infect Dis 2008;197:867-870.

6. Hirsch MS, Günthard HF, Schapiro JM, et al:: Antiretroviral drug resistance testing in adult HIV-1 infection: 2008 recommendations of an International AIDS Society-USA panel. Clin Infect Dis 2008;47:266-285.

7. Hirsch MS, Brun-Vézinet F, D'Aquila RT, et al.: Antiretroviral drug resistance testing in adult HIV-1 infection: Recommendations of an International AIDS Society-USA Panel. JAMA 2000;283:2417-2426.

8. Hirsch MS, Brun-Vézinet $\mathrm{F}$, Clotet $\mathrm{B}$, et al.: Antiretroviral drug resistance testing in adults infected with human immunodeficiency virus type 1: 2003 recommendations of an International AIDS Society-USA Panel. Clin Infect Dis 2003;37:113-128.

9. Wong KH, Chan KC, Cheng KL, Chan WK, Kam KM, and Lee SS: Establishing CD4 thresholds for highly active antiretroviral therapy initiation in a cohort of HIV-infected adult Chinese in Hong Kong. AIDS Patient Care STDS 2007;21:106-115.

10. Hong Kong Advisory Council on AIDS: Construction of the first set of core indicators (2003) for monitoring Hong Kong's AIDS programmes, 2004 [http://www.info.gov.hk/aids/ pdf/g136.pdf].

11. Scientific Committee on AIDS: Classification system for HIV infection and surveillance case definition for AIDS in adolescents and adults in Hong Kong. Hong Kong, 1995 [http://www.info.gov.hk/aids/pdf/g40.pdf].

12. Yam WC, Chen JH, Wong $\mathrm{KH}$, et al.: Clinical utility of genotyping resistance test on determining the mutation patterns in HIV-1 CRF01_AE and subtype B patients receiving antiretroviral therapy in Hong Kong. J Clin Virol 2006;35:454-457.

13. Chen JH, Wong KH, Chan $\mathrm{K}$, et al.: Evaluation of an in-house genotyping resistance test for HIV-1 drug resistance interpretation and genotyping. J Clin Virol 2007;39:125-131.

14. Ishizaki A, Cuong NH, Thuc PV, et al.: Profile of HIV type 1 infection and genotypic resistance mutations to antiretroviral drugs in treatment-naive HIV type 1-infected individuals in Hai Phong, Viet Nam. AIDS Res Hum Retroviruses 2009;25:175-182.

15. Kousiappa I, van de Vijver DA, Demetriades I, et al.: Genetic analysis of HIV type 1 strains from newly infected untreated patients in Cyprus: High genetic diversity and low prevalence of drug resistance. AIDS Res Hum Retroviruses 2009;25:23-35.
16. Tee KK, Kamarulzaman A, and Ng KP: Low prevalence of genotypic drug resistance mutations among antiretroviralnaive HIV type 1 patients in Malaysia. AIDS Res Hum Retroviruses 2006;22:121-124.

17. Choi JY, Kim EJ, Park YK, et al.: National survey for drugresistant variants in newly diagnosed antiretroviral drugnaive patients with HIV / AIDS in South Korea: 1999-2005. J Acquir Immune Defic Syndr 2008;49:237-242.

18. Yerly $\mathrm{S}$, von Wyl V, Ledergerber $\mathrm{B}$, et al.: Transmission of HIV-1 drug resistance in Switzerland: A 10-year molecular epidemiology survey. AIDS 2007;21:2223-2229.

19. Chaix ML, Descamps D, Wirden M, et al.: Stable frequency of HIV-1 transmitted drug resistance in patients at the time of transmitted infection over 1996-2006 in France. AIDS 2009; 23:717-724.

20. Wensing AM, van de Vijver DA, Angarano G, et al.: Prevalence of drug-resistant HIV-1 variants in untreated individuals in Europe: Implications for clinical management. J Infect Dis 2005;192:958-966.

21. Shet A, Berry L, Mohri H, et al.: Tracking the prevalence of transmitted antiretroviral drug-resistant HIV-1: A decade of experience. J Acquir Immune Defic Syndr 2006;41:439-446.

22. Fong OW, Ho CF, Fung LY, et al.: Determinants of adherence to highly active antiretroviral therapy (HAART) in Chinese HIV/AIDS patients. HIV Med 2003;4:133-138.

23. Yerly S, Vora S, Rizzardi P, et al.: Acute HIV infection: Impact on the spread of HIV and transmission of drug resistance. AIDS 2001;15:2287-2292.

24. Truong HM, Grant RM, McFarland W, et al.: Routine surveillance for the detection of acute and recent HIV infections and transmission of antiretroviral resistance. AIDS 2006;20: 2193-2197.

25. Grant RM, Hecht FM, Warmerdam M, et al.: Time trends in primary HIV-1 drug resistance among recently infected persons. JAMA 2002;288:181-188.

26. Bennett DE, Bertagnolio S, Sutherland D, et al.: The World Health Organization's global strategy for prevention and assessment of HIV drug resistance. Antivir Ther 2008; 13(Suppl 2):1-13.

27. Liu L, May S, Richman DD, et al.: Comparison of algorithms that interpret genotypic HIV-1 drug resistance to determine the prevalence of transmitted drug resistance. AIDS 2008;22:835-839.

28. Han X, Zhang M, Dai D, et al.: Genotypic resistance mutations to antiretroviral drugs in treatment-naive HIV/AIDS patients living in Liaoning Province, China: Baseline prevalence and subtype-specific difference. AIDS Res Hum Retroviruses 2007;23:357-364.

29. SPREAD programme: Transmission of drug-resistant HIV-1 in Europe remains limited to single classes. AIDS 2008;22: 625-635.

30. Pillay D, Green H, Matthias R, Dunn D, et al. and UK Collaborative Group on HIV Drug Resistance: Estimating HIV-1 drug resistance in antiretroviral-treated individuals in the United Kingdom. J Infect Dis 2005;192:967-973.

Address correspondence to: K.H. Wong

Special Preventive Programme Centre for Health Protection Department of Health

9/F, Kowloen Bay Health Centre

9, Kai Yan Street Kowloon, Hong Kong

E-mail: khwong@dh.gov.hk 
